\title{
Removal of Motion Noise from Surface-electromyography Signal Using Wavelet Adaptive Filter
}

\author{
Wang Fei ${ }^{1, a}$, Qiao Xiao-yan², b \\ ${ }^{1}$ College of Physics and Electronics Engineering, Shanxi University, Taiyuan, China, 030006 \\ ${ }^{2}$ College of Physics and Electronics Engineering, Shanxi University, Taiyuan, China, 030006 \\ aemail:457112458@qq.com, bemail: xyqiao@sxu.edu.cn
}

\section{Keyword: Surface-electromyography signal; Wavelet Transform; Adaptive Filter; Motion Noise}

\begin{abstract}
Surface-electromyography (sEMG) is a kind of weak physiological signals. During the sEMG signals measurement, the motion noise caused by body movement can easily affect measurement accuracy. In order to prevent the noise, we designed a method to adaptive noise cancellation combined with wavelet transform for sEMG signal filter. Through the decomposition and reconstitution of the Surface-electromyography signal using wavelet transform, we extracted motion interference signal, as the reference input signal of adaptive noise canceller, to offset motion noise of the original sEMG signal. Without the loss of useful sEMG information, this method can suppress motion noise interference and improve the SNR of the measurement system. It laid a solid foundation for EMG feature extraction and pattern recognition.
\end{abstract}

\section{Introduction}

Electromyographic(EMG) signal, which is the superposition on motor unit action potential (MUAP) from many muscle fibers in time and space, can reflect the neuromuscular activity to a certain extent. EMG amplitude is commonly $100 \sim 2000 \mu \mathrm{V}$, frequency range 20 to $500 \mathrm{~Hz}$, main energy concentrated around $20 \sim 150 \mathrm{~Hz}[1]$. Since the EMG signal is faint, it always is interfered with motion noise in measurement [2]. EMG signal is collected by surface electrodes fixed on skin. Because of electrode contacted with skin by electrolyte solution, such as electrode jelly or sweat, it can form a metal- electrolyte solution interface. Therefore, the electrode potentials are generated for chemical reaction on the interface. During the electromyographic acquisition, it always has strenuous exercise. This leads to electrode potential change with the contact area of wireless sensor and skin. It also can change with the polarization of the input bias current of amplifying circuit. The change's specific form is sEMG happened low frequency drift. This drift is called motion noise [3]. It mainly concentrates below $20 \mathrm{~Hz}$. When we collect sEMG has huge body movement, this problem is more grievous [4].

Now, we usually uses the digital filter eliminated motion noise, which is using high-pass digital filter eliminated frequency under $20 \mathrm{~Hz}$. Using this method we will eliminate noise and sEMG signal on the same frequency band. That makes signal information incomplete [5]. Using Wavelet Transform (WT) eliminated motion noise also faces the same question. However, under the condition of no signal features of prior knowledge, the adaptive filter has better performance. It's difficult to get reference signal of motion noise. It can influent motion noise elimination [6]. So, we combined wavelet transform with adaptive filter. We use this method as the sEMG preprocessing to remove the motion noise. Firstly, we use the multi-resolvability feature of wavelet transform to decompose sEMG signal and reconstruct motion noise. Then motion noise will be regard as the reference signal of adaptive filter. Under the condition of not loss the useful part of sEMG signal, we suppressed motion noise influence. 


\section{2 .Algorithm theory}

\section{1 wavelet transform}

Wavelet transform is a multi-resolution time-frequency analysis method. Because of its excellent time-frequency characteristics, it has been widely used in analysing and denoising the nonstationary time-varying signal.

If function $\psi(\mathrm{t}) \in L^{2}(R)$ satisfy $\int_{R} \psi(\mathrm{t}) d t=0$, it is defined wavelet function. After stretching and translation, we got $\psi_{a . b}=|a|^{-\frac{1}{2}} \psi\left(\frac{t-b}{a}\right)$. Where, a is scale factor, and $\mathrm{b}$ is translation factor. To continuous signal $f(t)$, wavelet transform and its inverse transform are:

$$
\begin{aligned}
& W f(a, b)=\left\langle f, \psi_{a, b}\right\rangle=\int_{R} f(t) \overline{\psi_{a, b}(t)} \mathrm{dt} \\
& f(t)=\frac{1}{c_{\psi}} \iint_{R} W f(a, b) \psi_{a, b}(t) d a d b / a^{2}
\end{aligned}
$$

To the discrete $\operatorname{signal} f(n)$, choosing $a=a_{0}^{j}, b=n b_{0} a_{0}^{j}\left(a_{0}>1, b_{0} \neq 0, j \in Z\right)$. Then, discrete wavelet transform satisfy following equation:

$$
W^{j} f(n)=W f(a, b)=a_{0}^{-\frac{1}{2}} \sum_{k} f(k) \overline{\psi\left(a_{0}^{j} k-n b_{0}\right)}
$$

Discrete binary wavelet transform of $f(n)$ is:

$$
\begin{aligned}
& f^{j}(n)=\sum_{k} h(k) f^{j-1}\left(\mathrm{n}-2^{j-1} \mathrm{k}\right) \\
& W^{j} f(n)=\sum_{k} g(k) f^{j-1}\left(n-2^{j-1} k\right)
\end{aligned}
$$

Where, $h(k)$ and $g(k)$ respectively is the coefficient of orthogonal digital filter $H(\omega)$ and $(\omega)$. Wavelet $G(\omega)$. Wavelet multi-resolution analysis is based on Matllat algorithm. Matllat algorithm uses a set of orthogonal digital filter to process the signal. Wavelet transform decompose the original signal into approximation signal and detail signal. The wavelet reconstruction is the inverse operation of wavelet decomposition.

\subsection{Adaptive filtering}

Adaptive filter can adjust the best weight and output characteristic by itself. As a classical adaptive filter, adaptive noise canceller is based on adaptive filtering algorithm. It is using the difference between original signal and reference signal to eliminate noise and extract pure EMG signal. LMS algorithm is used to adjust adaptive noise canceller weight. The function is following.

$$
W_{j+1}=W_{j}+2 \mu e_{j} X_{j}
$$$$
e_{j}=d_{j}-W_{j}^{T} X_{j}
$$

In the function, $W_{j}$ is the weight, $d_{j}$ is desired signal, $X_{j}$ is the input signal, $e_{j}$ is the error signal, $\mu$ is the step factor.

Figure 1 is principle chart of adaptive noise canceller. It has original signal input terminal and reference signal input terminal. Reference signal correlated with noise signal and uncorrelated with EMG signal. LMS adaptive algorithm is using error signal $\left(e_{j}\right)$ to control weight $\left(W_{j}\right)$. In the condition of minimum mean square error, when the filter output $y_{T}$ approximately equal noise signal $n_{T}$, error signal $e_{T}$ is approximately equal ideal signal $s_{T}$. 


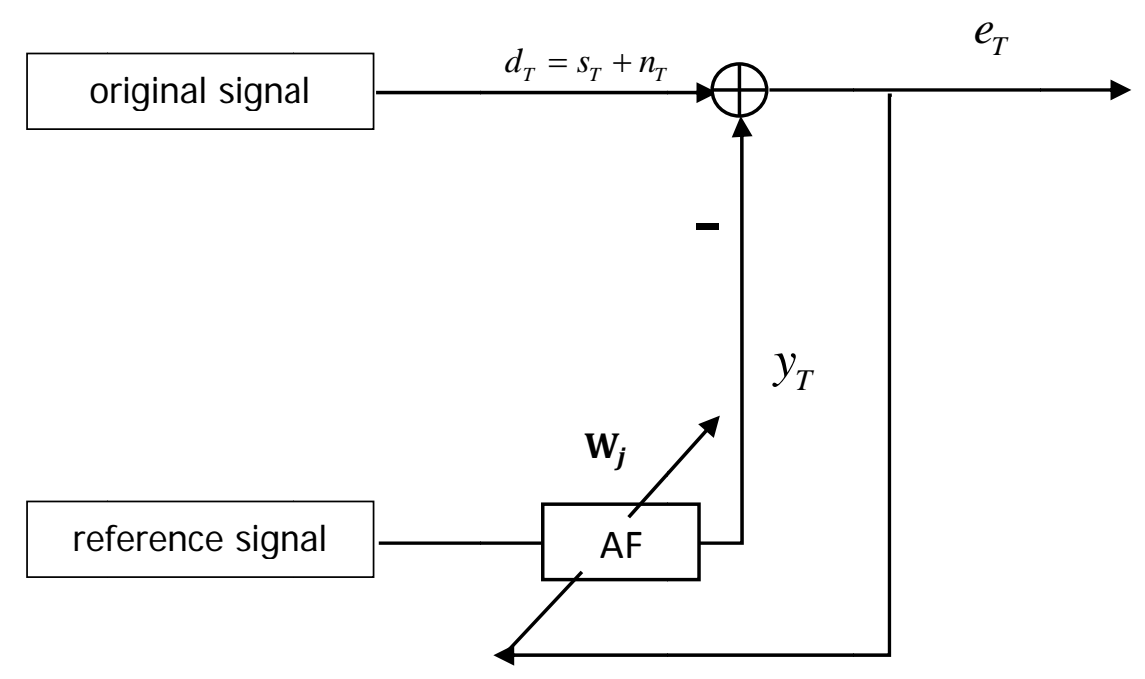

Fig.1 Principle chart of adaptive noise canceller

\section{Signal acquisition and filtering}

\subsection{Collecting Original Signal}

We choose 20-22 years old healthy man as experimental subject. Experimental subject is demanded not do strenuous exercise before experiment. In order to acquisition clean EMG and reducing impedance between skin and electrode sensors. Before the experiment, experimental subject target muscle should be cleaned cuticle with alcohol. Experiment captures EMG signal of brachioradialis, flexor carpi ulnaris and flexor carpi radialis under the hand enstrophe, hand ectropion, wrist intorsion and wrist extorsion. Each motion repeats 15 times, each time maintains $5 \mathrm{~s}$. Wireless transceiver transfers every motion EMG to sEMG measuring instrument. We use USB connector to transfer EMG to computer. We save and analyze it on computer.

The experiment uses Delsys as EMG acquisition system. It is the 16 channels EMG acquisition system made by Noraxon. This system has high reliability and easier operating. Delay between electrode sensors is smaller than $0.5 \mathrm{~ms}$. It has more resistant to interference and noise. Baseline noise is smaller than $750 \mathrm{nv}$, common mode rejection ratio is larger than $80 \mathrm{~dB}$ and sampling rate is $2000 \mathrm{~Hz}$. Figure 2 is the EMG acquisition system principle chart.

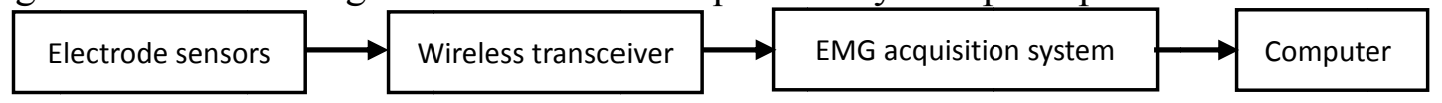

Fig.2 EMG acquisition system principle chart

sEMG signal has the same analytical method. This paper chooses hand enstrophe brachioradialis EMG as example.

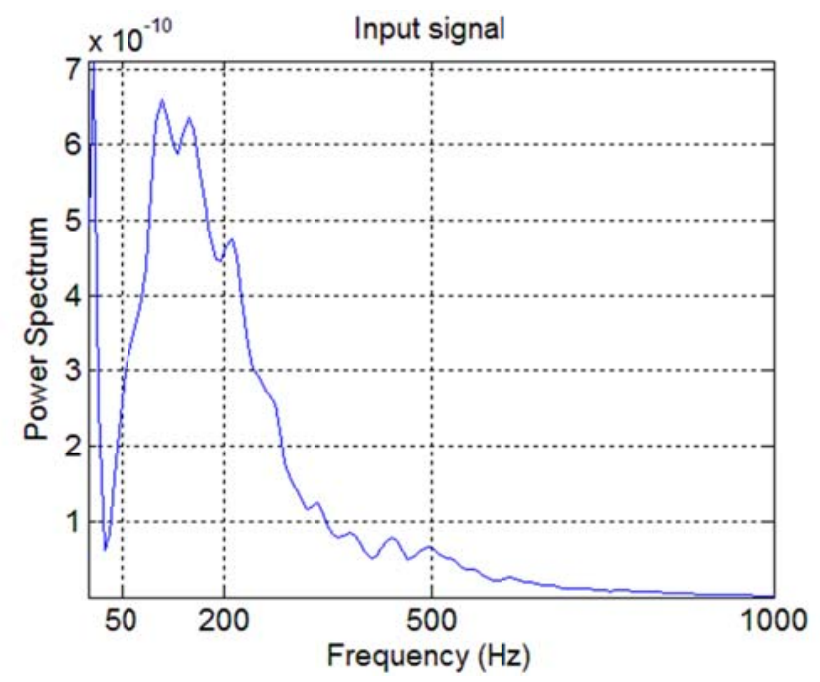

Fig.3 Power spectrum of the original EMG signal 


\subsection{Filter motion noise}

Because of $\mathrm{dbN}$ wavelet orthogonality, we choose db3 wavelet as wavelet basis function decomposed the original signal into 7 th order sub-signal. Sub-signal frequency band is between 0 and $16 \mathrm{~Hz}$. Then, we get a slowly varying time domain signal by reconstructed 7 th order wavelet coefficients as reference signal. Its frequency main concentrate on $0 \sim 20 \mathrm{~Hz}$. Reference signal is the motion noise. Finally, we used wavelet adaptive filter to eliminate motion noise.

Step factor $\mu$ can directly affect the filter performance. Parametric $\mu$ control stability and the convergence rate of system. Incorrect value of $\mu$ can cause local convergence. When the value is too large, convergence rate is too fast to get the best weight. On the contrary, convergence rate is more stable and accurate. In this experiment, we choose 0.0005 as the value of $\mu$.

\section{Analysis}

Figure 4 is the original signal motion noise. It's extracted by wavelet decomposition and reconstruction. Figure (a) is the motion noise time domain waveform. Figure (b) is the motion noise frequency domain waveform. It shows that the motion noise can be extracted by wavelet decomposition and reconstruction.

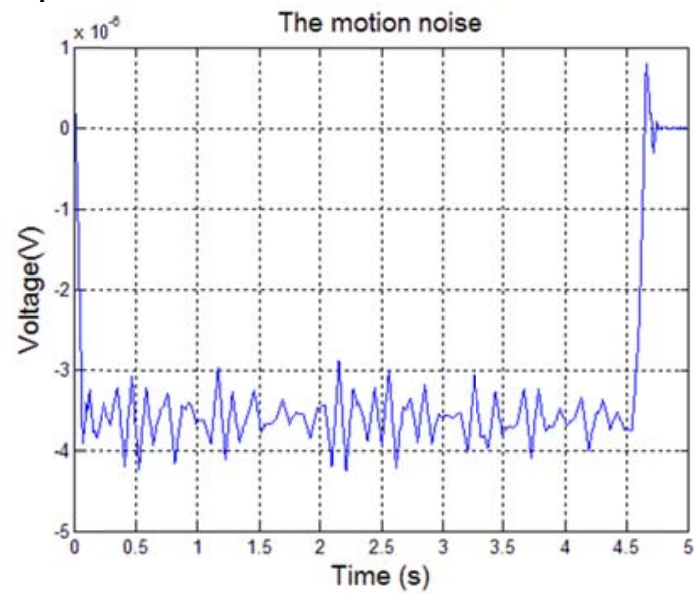

(a)

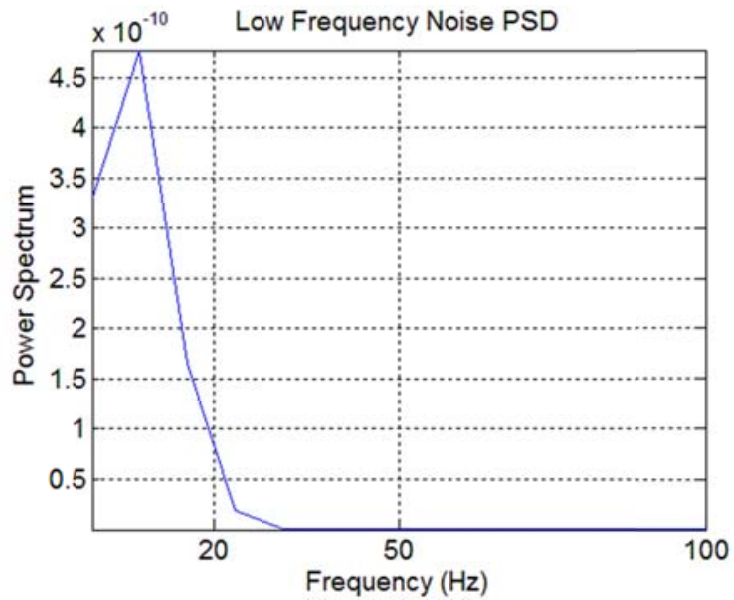

(b)

Fig.4 The motion noise

(a) The time domain waveform of motion noise

(b) The frequency domain waveform of motion noise

Figure 5 is pure EMG signal power spectrum. Compared figure 5 with figure 3, it has shown that adaptive filter can suppresse the motion noise and save the low frequency components of EMG signal and improve SNR at the same time.

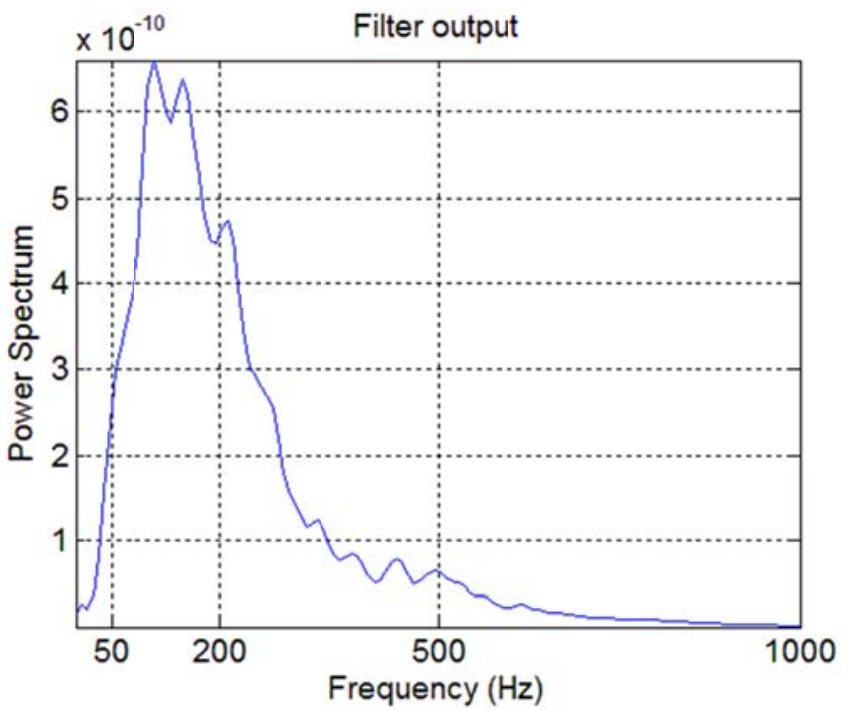

Fig.5 Power spectrum of EMG signal after wavelet adaptive filter 


\section{Conclusion}

Under the condition of not loss the useful part of sEMG signal, wavelet adaptive filter suppressed motion noise, got pure sEMG signal and improved the SNR of the signal. It laid a solid foundation for feature extraction and pattern recognition.

\section{References}

[1] CHENG Xiu-fang, HOU Wei-min, XU Wen-mo. The research For sEMG Processing Used For Biological Model [J]. Journal of Hebei United University ( Natural Science Edition),2014,01:61-65.

[2] Sardar D., Basu D., Bhattacharyya S., Konar A., Khasnobish A., Tibarewala D.N., Janarthanan R., "Embedded realisation of amplitude-phase adaptive filter for bio-potential signals," 2014 International Conference on Control, Instrumentation, Energy and Communication (CIEC). 2014, pp.593-597.

[3]Zhangyan Zhao. Several Key Technologies in Surface Electromyography Signal Detection \& Processing [D].University of Science and Technology of Chain,2010.

[4] XIONG Ping, TANG Jian. Surface EMG signal preamplifier system [J]. Computer Engineering and Applications,2015, 51 (1): 218-222.

[5]Pu Liu, Lukai Liu, Clancy E.A., "Influence of Joint Angle on EMG-Torque Model During Constant-Posture, Torque-Varying Contractions," IEEE Transactions on Neural Systems and Rehabilitation Engineering. 2015, vol.23, no.6, pp.1039-1046.

[6]Hashim F.R., Soraghan J.J., Petropoulakis L., Daud N.G.N., "EMG cancellation from ECG signals using modified NLMS adaptive filters," 2014 IEEE Conference on Biomedical Engineering and Sciences (IECBES). 2014, pp.735-739. 